\title{
GLADIOLUS DALENII (IRIDACEAE, CROCOIDEAE) NATURALIZADA EN MISIONES, ARGENTINA
}

\author{
Gladiolus dalenii (Iridaceae, Crocoideae) naturalized in Misiones, Argentina
}

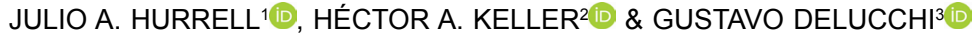

\begin{abstract}
Resumen: En este trabajo se menciona por primera vez para la Argentina la presencia de Gladiolus dalenii (Iridaceae, Crocoideae) como especie naturalizada en la provincia de Misiones. Se incluye la descripción de la especie, sinonimia, iconografía, distribución geográfica, fenología, polinización, dispersión, nombres vernáculos, usos, material de referencia y comentarios sobre su naturalización. También se provee una clave para distinguir a $G$. dalenii de $G$. communis y G. tristis, las otras especies del género citadas con anterioridad como naturalizadas en el país. Además, se incluyen comentarios sobre híbridos de flores grandes escapados de cultivo en la provincia de Buenos Aires.
\end{abstract}

Palabras clave: Argentina, geófitas, Iridaceae, naturalización, ornamentales.

\begin{abstract}
Summary: In this work, the presence of naturalized Gladiolus dalenii (Iridaceae, Crocoideae) is reported for the first time for Argentina (Misiones province). The species description, synonyms, iconography, geographical distribution, phenology, pollination, dispersal, local names, uses, reference materials, and comments about its naturalization are provided. This study also presents a key to distinguish $G$. dalenii from $G$. communis and G. tristis, the other species of the genus already reported as naturalized in the country. Finally, findings of Gladiolus hybrids of large flowers escaped from cultivation in Buenos Aires province are commented.
\end{abstract}

Key words: Argentina, geophytes, Iridaceae, naturalization, ornamentals.

\section{Introducción}

En esta contribución se menciona por primera vez para la Argentina la presencia de Gladiolus dalenii Van Geel (Iridaceae, Crocoideae), especie naturalizada en la provincia de Misiones. Con esta nueva cita, el total de Iridaceae de la Flora Argentina asciende a 25 géneros y 102 especies, de los cuales 7 géneros y 12 especies son naturalizados. Dentro de estos últimos, la subfamilia Crocoideae incluye 6 géneros y 10 especies, entre estas, 3 especies pertenecen al género Gladiolus Tourn. ex L.: G. dalenii y las anteriormente citadas G. communis L. y $G$. tristis L. (Hurrell \& Delucchi, 2005; Delucchi \& Hurrell, 2009).

Gladiolus comprende aproximadamente 250-265 especies de la región Mediterránea, Europa, sudoeste de Asia hasta Afganistán, África cálida y Sudáfrica, donde se halla la mayor concentración de especies (Goldblatt,

${ }^{1}$ Laboratorio de Etnobotánica y Botánica Aplicada, Facultad de Ciencias Naturales y Museo, Universidad Nacional de La Plata. Calle 64 nro. 3, 1900-La Plata. Investigador CONICET, Argentina. E-mail: juliohurrell@gmail.com

${ }^{2}$ Facultad de Ciencias Forestales, Universidad Nacional de Misiones, Instituto de Botánica del Nordeste, Casilla de Correo 209, 3400-Corrientes. Investigador CONICET, Argentina. E-mail: kellerhector@hotmail.com

${ }^{3}$ Facultad de Ciencias Naturales y Museo, Universidad Nacional de La Plata. Paseo del Bosque s/nro. 1900-La Plata, Argentina. E-mail: delucchi@fcnym.unlp.edu.ar 
1996a, b, 2002; Goldblatt \& Manning, 1998; Goldblatt et al., 1998, 2008; Hamilton, 2005; Cantor \& Tolety, 2011; Yeo, 2011; Singh et al., 2018; Hyde et al., 2019). El nombre genérico proviene del latín gladius, "espada" y el diminutivo -ulus, espada corta difundida en la Antigua Roma, que alude a sus hojas ensiformes; por ese mismo carácter los gladiolos también eran reconocidos en la Grecia clásica, donde recibían el nombre de xiphium (de xiphos, "espada"). Los gladiolos se conocen en Anatolia (Turquía) hace más de 2000 años y algunas especies se han cultivado por más de 500 años en Europa (Cantor \& Tolety, 2011).

Desde principios del siglo XIX, diversas especies sudafricanas se introdujeron en Europa, y hacia 1910 los floricultores desarrollaron cruzamientos entre cuatro o cinco de esas especies, entre ellas $G$. dalenii, con posterior selección, obteniendo híbridos denominados "gladiolos de flores grandes" o "gladiolos de los floristas", de muy amplia difusión (Goldblatt, 2002; Cantor \& Tolety, 2011; Singh et al., 2018). Estos híbridos no se pueden identificar con ninguna de las especies indígenas del género, por lo que se suelen agrupar bajo los nombres hortícolas de G. $\times$ grandiflorus Hort. (no G. grandiflorus Andrews, Bot. Repos. 2: tab. 118. 1800) o G. $\times$ hybridus Hort. (Anderson \& Park, 1989). En la actualidad, Gladiolus constituye uno de los principales cultivos de geófitas ornamentales en el mundo, apreciadas para jardines y, en especial, para flores de corte (Cantor \& Tolety, 2011).

Gladiolus dalenii se ha introducido y naturalizado en diversos países de zonas cálidas y templadas, y a menudo deviene invasora y/o maleza, en términos de la ecología de las invasiones biológicas (Rejmánek, 2000; Richardson et al., 2000, 2010, 2011; Pyšek et al., 2002, 2004; Pyšek \& Richardson, 2006; Richardson \& Pyšek, 2012; Randall, 2017). En este contexto, el cultivo de especies ornamentales constituye una de las principales vías de ingreso de especies exóticas que pueden naturalizarse (Reichard \& White, 2001; Groves et al., 2005; Kowarik, 2005; Dehnen-Schmutz et al., 2007; Foxcroft et al., 2008). En el marco del proceso de naturalización de las especies exóticas que pueden devenir invasoras, como es el caso de $G$. dalenii en otros países, el primer registro de la especie como naturalizada en la Argentina resulta relevante para evaluar su posible expansión en el futuro.

En este trabajo se incluye la descripción de $G$. dalenii, sinonimia (se seleccionaron los sinónimos más frecuentes que figuran en la bibliografía de nuestro país), iconografía, distribución geográfica, fenología, polinización, dispersión, nombres vernáculos, usos, material de referencia, una clave dicotómica para distinguir a $G$. dalenii de $G$. communis y $G$. tristis, las otras especies del género naturalizadas en el país, comentarios sobre los gladiolos híbridos hallados escapados de cultivo en la provincia de Buenos Aires, y una discusión sobre la naturalización de G. dalenii.

\section{Resultados}

Gladiolus dalenii Van Geel, Sert. Bot. 2: 18. 1829.

Typus: Sudáfrica. Ilustración tab. 18, loc. cit. (Tropicos, 2018).

Gladiolus psittacinus Hook., Bot. Mag. 57: tab. 3032. 1830; G. natalensis Reinw. ex Hook., Bot. Mag. 58: sub tab. 3084. 1831, nom. superfl.; $G$. dracocephalus Hook. f., Bot. Mag. 97: tab. 5884. 1871; G. primulinus Baker, Gard. Chron. III, 8: 122. 1890 .

Iconografia: Van Geel, 1829 (loc. cit.); Hooker, 1830 (loc. cit.); Hooker f., 1871 (loc. cit.); Goldblatt, 1989: 253, fig. 6; Goldblatt, 1991: 41, pl. 6, 4; Goldblatt, 1996a: 69; Goldblatt, 2002: 408.

Hierbas perennes de 0,5-1,5 m alt. Cormos ovoides de 1,5-3 cm diám., con túnicas castañorojizas, coriáceas a membranáceas, capa externa fibrosa a irregularmente desgarrada en general, con numerosos cormos hijos pequeños en torno a la base. Tallos floríferos simples. Hojas simultáneas con la floración, erectas, 4-6(-7), al menos 2 inferiores, \pm basales, de $30-60 \times 0,5-5 \mathrm{~cm}$, ensiformes, nervio medio y márgenes moderadamente gruesos, hojas superiores 1-2, caulinares, envainadoras al menos la mitad de su longitud. Inflorescencias en espigas terminales, erectas, 2-7(-14) floras; brácteas de 3-7 cm long., verdes, la interna ligeramente más corta. Flores de $6-8 \mathrm{~cm}$ long., zigomorfas, infundibuliformes, inodoras, tubo del perianto de 2,5-4,5 cm long., \pm cilíndrico, 
curvado hacia afuera en su mitad superior. Tépalos $3+3$, desiguales, rojos o anaranjadorojizos, con mancha amarilla en los tépalos externos (inferiores), o bien amarillos, lisos o con rayas rojizas a castañas en tépalos internos (superiores); tépalos internos de 3,5-5 $\times 2-3 \mathrm{~cm}$, anchamente elípticos u obovados, el dorsal mayor que los laterales, horizontal o curvado hacia abajo, los laterales subiguales, el más largo dirigido hacia adelante, a menudo curvado hacia afuera en la porción distal; tépalos externos de $2-3 \mathrm{~cm} \times 0,8-1,2 \mathrm{~cm}$, curvados hacia abajo, el inferior más largo $\mathrm{y}$ angosto que los laterales. Estambres 3, unilaterales, exsertos; filamentos de 2,5-3 cm long.; anteras de 1,2-1,6 cm long., amarillo pálidas. Ovario ovoide, nectarios septales, estilo exerto con 3 ramas estilares de 0,4-0,6 $\mathrm{cm}$ long. Fruto cápsula elipsoide u obovoide, de 1,8-3,5 × 1,2-1,4 cm. Semillas aladas, de $0,8-1,2 \times 0,5-1 \mathrm{~cm}$, castaño claras, lustrosas. $2 n$ $=30$ (Goldblatt, 1989); 45, 60, 75 (Goldblatt, 1971); 60, 90 (Goldblatt et al., 1993); 50, 54, 56 (Kalaiselvi, 2002); 80 (Darlington \& Wylie, 1955).

Distribución. África tropical subsahariana, Sudáfrica, Madagascar y sudoeste de la Península Arábiga, es una de las especies del género con distribución más amplia. Crece en bosques abiertos y pastizales, también en humedales y zonas semiáridas con suelos rocosos; en Sudáfrica se encuentra hasta los 2000 m s.n.m., en zonas de lluvias estivales (Goldblatt, 1991, 1993, 1996a, b; Goldblatt \& Manning, 1998; Lim, 2014; Govaerts \& Barker, 2018). Según Goldblatt (1993), se ha registrado, al menos en el oeste de África, su cultivo en huertos de zonas boscosas, introducida desde las áreas de sabanas. En este marco, dicho autor comenta: “¿Cuánto de su distribución notablemente amplia se debe a actividades humanas deliberadas? Tal vez nunca se sepa".

Gladiolus dalenii fue introducida en Europa hacia 1825 por Cornelius Dalen (1766-1852), botánico y médico holandés, director del Jardín Botánico de Rotterdam, a quien se dedicó la especie. Se ha naturalizado en los Estados Unidos (Alabama, Florida, Luisiana, Hawái), Azores, Reunión, India, Nueva Zelanda y Australia (Nueva Gales del Sur). Esta especie a menudo deviene invasora y/o maleza (Healy
\& Edgar, 1980; Goldblatt, 2002; Hosking et al., 2011; Franck et al., 2016; Randall, 2017).

En la Argentina, el cultivo de esta especie fue señalado por Dimitri (1987) bajo el nombre de $G$. primulinus Baker, plantas de flores amarillas. En la provincia de Misiones crecen de forma espontánea plantas de flores rojas o anaranjado-rojizas con mancha amarilla en los tépalos externos (Fig. 1); estas son frecuentes en ambientes alterados desde hace varios años en la provincia (Irma S. Insaurralde, com. pers.), principalmente en bordes de rutas y caminos asfaltados; también en cultivos de yerba mate, donde se hallaron poblaciones densas en caminos terrados. Los bordes de caminos y rutas funcionan como "corredores" que facilitan la propagación de especies exóticas introducidas que se naturalizan y eventualmente pueden devenir invasoras (Trombulak \& Frissell, 2000).

Fenología, polinización y dispersión. Florece desde la primavera hasta el otoño. La polinización en su área de origen es realizada por Passeriformes Nectariniidae atraídos por su copioso néctar (Goldblatt \& Manning, 2002, 2006; Valente et al., 2012). Las semillas aladas tienen dispersión anemócora (Goldblatt et al., 1998), y asimismo las plantas se expanden por propagación vegetativa, por medio de sus cormos; a menudo estos pueden ser ingeridos por cerdos salvajes en su área original o descartados de los cultivos y trasladados con los movimientos de tierra y desechos de jardines (Hosking et al., 2011; Lim, 2014). Este mecanismo de propagación vegetativa involuntaria se ha mencionado para otras especies de geófitas naturalizadas en el país (Hurrell \& Delucchi, 2005, 2007a, b; Hurrell et al. 2009, 2010, 2019).

Nombres vernáculos. "Gladiolo", "gladiolo africano", "gladiolo sudafricano".

Usos. Se cultiva ampliamente como ornamental; no obstante, su principal valor es su rol como progenitor en los cruzamientos originales que desarrollaron los gladiolos híbridos de flores grandes (Goldblatt, 1993, 2002). Además, varios cultivares de G. dalenii se difundieron en Europa desde el siglo XIX, para jardines y flores de corte. Estos híbridos 


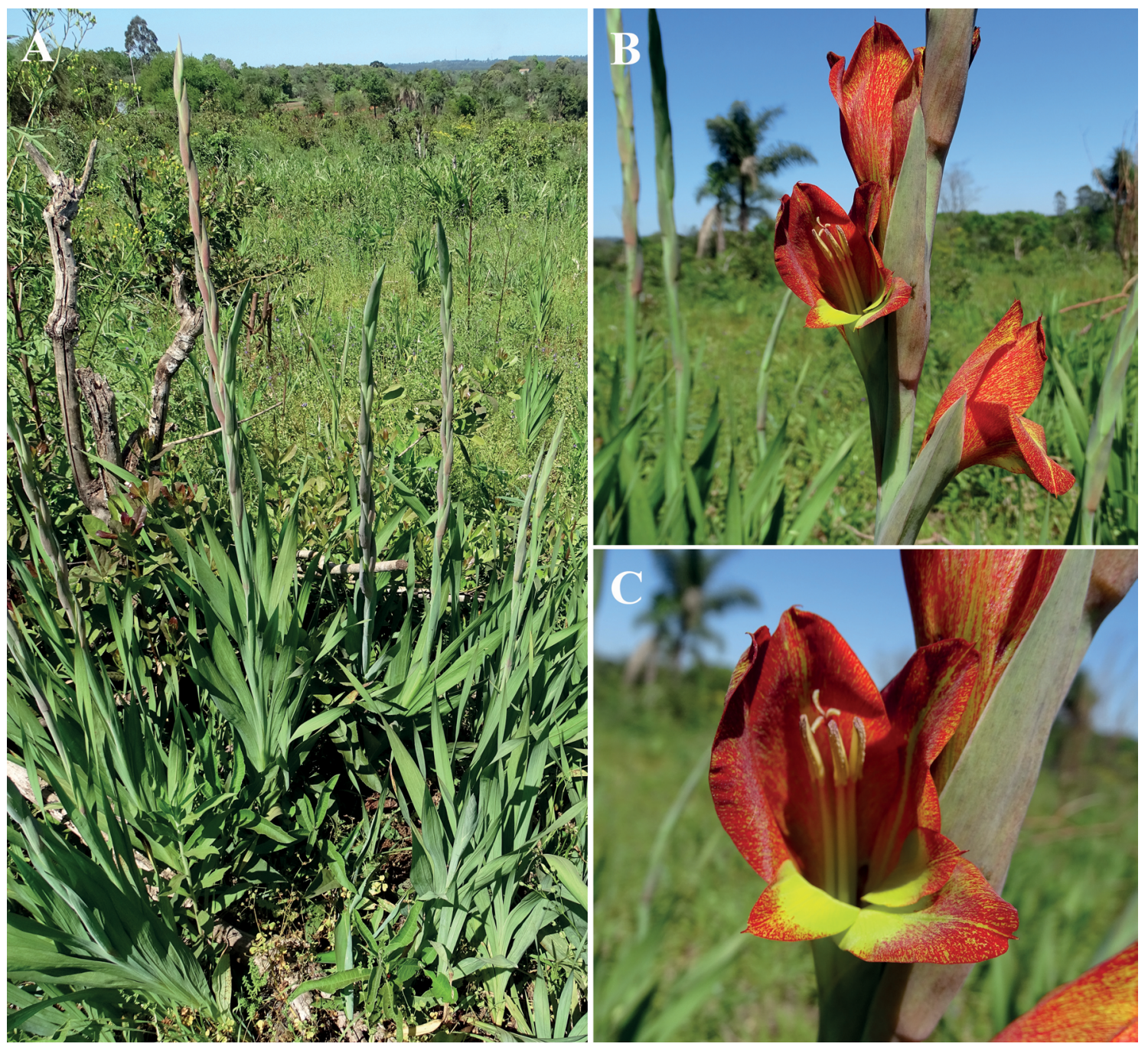

Fig. 1. Gladiolus dalenii. Plantas naturalizadas en Misiones. A: Plantas con espigas en desarrollo. B: Detalle de flores en la espiga. C: Detalle de la flor (Fotos: H. A. Keller).

Fig. 1. Gladiolus dalenii. Naturalized plants in Misiones. A: Plants with developing spikes. B: Detail of flowers in the spike. C: Detail of the flower (Photos: H. A. Keller).

toleran las bajas temperaturas y prosperan mejor en suelos frescos, bien drenados, arenosos o franco-arenosos, con $\mathrm{pH}$ neutro o ligeramente ácido, en sitios asoleados, protegidos (Yeo, 2011; Lim, 2014). En cultivo se reproduce por semillas, aunque mayormente se propaga por cormos (Fig. 2).

Gladiolus dalenii es una de las pocas especies del género valorada por sus usos no ornamentales, como el medicinal y el alimentario (Goldblatt, 1993). A partir de las hojas se obtienen fibras empleadas para encordados y trenzados (Lim, 2014). En África tropical, los cormos amiláceos se hierven y lixivian en agua antes de ser consumidos (Goldblatt, 1993). Las flores, una vez removidas las anteras, se consumen frescas en ensaladas o cocidas como verdura, y el néctar, en ocasiones, es bebido principalmente por los niños (Facciola, 1998).

Los cormos se emplean en medicina tradicional de diversos pueblos africanos, por lo común en maceraciones o decocciones, como antiepiléptico, sedante, anti-insomnio, antidiarreico, antidisentérico, antiestreñimiento, antiespasmódico, vermífugo, antídoto, antitusivo, antioftálmico, vulnerario, 

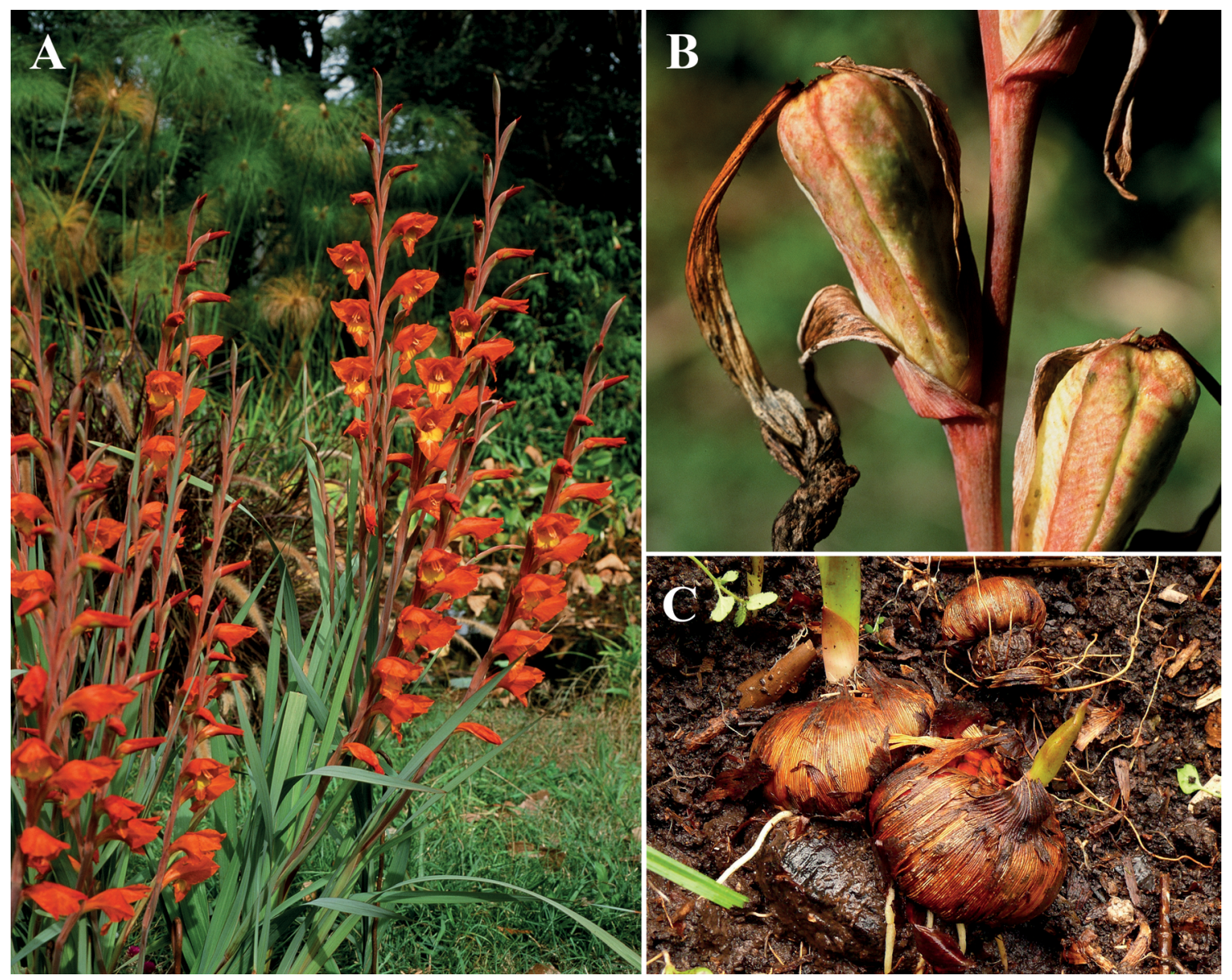

Fig. 2. Gladiolus dalenii. Plantas cultivadas en el Jardín Botánico "Lucien Hauman”, Facultad de Agronomía, UBA, Ciudad Autónoma de Buenos Aires. A: Plantas en floración. B: Detalle de los frutos. C: Detalle de los cormos (Fotos: D. H. Bazzano).

Fig. 2. Gladiolus dalenii. Plants cultivated in the "Lucien Hauman" Botanical Garden, Faculty of Agronomy, UBA, Autonomous City of Buenos Aires. A: Flowering plants. B: Detail of the fruits. C: Detail of the corms (Photos: D. H. Bazzano).

para tratar la esquizofrenia y otros desórdenes psicóticos, dolores de cabeza y de oído, dismenorrea, cólera, reumatismo, lumbago y artritis; además, el humo de los cormos al ser quemados se inhala para tratar los resfríos (Goldblatt, 1993; Fawole et al., 2009; Gbadamosi, 2012; Ngoupaye et al., 2013a, b, 2014; Lim, 2014). En Nigeria, los cormos se utilizan para combatir la gonorrea y la candidiasis vulvo-vaginal (Gbadamosi \& Egunyomi, 2014), y la tintura de las semillas se emplea para combatir la infertilidad femenina (Sharaibi et al., 2017). En Benín, los cormos en decocción o pulverizados se consideran antidiabéticos (Lawin et al., 2015). En Kenia, los cormos en distintas preparaciones sirven para tratar la meningitis, malaria, diarrea, úlceras e infecciones oportunistas vinculadas al HIV (Odhiambo et al., 2010, 2011). En Uganda, la maceración de raíces y hojas se usa como febrífugo y antimalarial (Opio et al., 2017). En Madagascar, una pomada a base de los cormos sirve para tratar abscesos (Lim, 2014). En la India, fuera de su área de origen, las raíces se emplean para casos de mordeduras de serpientes (Pullaiah et al., 2016).

En el ámbito académico se ha evaluado su actividad biológica y sus efectos antidepresivo, sedante, anticonvulsivo (Ngoupaye et al., 2013a, b; 2014), ansiolítico (Fotsing et al., 
2017), potenciador cognitivo: anti-amnesia (Ngoupaye et al., 2017), anti-Candida (Giordani et al., 2015), antifúngico (Odhiambo et al., 2010), antibacteriano (Gbadamosi, 2012), antiinflamatorio (Fawole et al., 2009).

En medicina veterinaria, los cormos se usan en mezclas para tratar dolencias vaginales del ganado, y la savia de los cormos se aplica como gotas nasales (Lim, 2014). Las raíces se utilizan como contraceptivo y abortivo (Quattrocchi, 2016).

Material examinado: ARGENTINA. Misiones: Dep. Montecarlo, acceso a Puerto Piray, ruta nacional 12, capuera, 12-X-2008, fl., H. A. Keller 6408(CTES). Dep. Eldorado, Colonia Victoria, inmediaciones del peaje, 26²0'09,3'”S - 54³7'08,7' W, 25-IX-2016, fl., H. A. Keller \& J. L. Rojas 13415 (CTES).

Material adicional cultivado: ARGENTINA. Ciudad Autónoma de Buenos Aires: Facultad de Agronomía, Jardín Botánico "Lucien Hauman", 15-X-2004, fl., J. A. Hurrell \& D. H. Bazzano 5840 (BAB).

Obs. 1. Gladiolus dalenii se diferencia de G. communis y G. tristis, las otras especies del género naturalizadas en nuestro país, mediante los caracteres de la siguiente clave.

\section{Clave para las especies naturalizadas de Gladiolus en Argentina}

1. Láminas foliares cruciformes en sección transversal. Flores muy perfumadas, blancas a cremosas con tintes purpúreos o amarilloverdosos. G. tristis

1'. Láminas foliares planas. Flores no perfumadas, purpúreo-rojizas, anaranjado-rojizas o rojas.

2. Espigas 10-20 floras. Tubo del perianto de 1-1,5 $\mathrm{cm}$ long. Tépalos externos en general purpúreorojizos con raya media blanca angosta. Cápsula oblonga hasta de 2,4 cm long. Semillas de 0,4$0,6 \mathrm{~cm}$ long. G. communis

2'. Espigas 2-7(-14) floras. Tubo del perianto de 2,5-4,5 cm long. Tépalos externos rojos o anaranjado-rojizos con mancha amarilla, o bien amarillos. Cápsula elipsoide u obovoide hasta de 3,5 cm long. Semillas de 0,8-1,2 cm long. ......
Obs. 2. Se han citado ocho especies de Gladiolus cultivadas en la Argentina, cuatro de estas son especies botánicas reconocidas: G. communis, G. dalenii, G. tristis (Dimitri, 1987) y G. murielae Kelway (Hurrell et al., 2005); las otras cuatro especies son híbridos de flores grandes: $G . \times$ colvillei Sweet, $G$. $\times$ gandavensis Van Houtte, G. $\times$ lemoinei Baker y $G$. $\times$ nanceianus Baker (Dimitri, 1987), todos ellos considerados nombres no resueltos (The Plant List, 2013). Gladiolus $\times$ gandavensis, uno de los híbridos más difundidos desde su origen en 1837 hasta nuestros días, proviene del cruzamiento entre $G$. dalenii y $G$. oppositiflorus Herb. (Mukhopadhyay, 1995; Yeo, 2011). Según Goldblatt (2002) este y otros híbridos se expanden a menudo por vía vegetativa en las cercanías de los jardines y perduran algunos años escapados de cultivo en zonas templado-cálidas. Sin embargo, por lo general no muestran evidencias de su naturalización en la vegetación espontánea adyacente. En esta situación se hallan los ejemplares que corresponden al híbrido $G$. $\times$ gandavensis hallados en la provincia de Buenos Aires, en los partidos de La Plata: Gonnet, alrededores de jardín, plantas que persisten varios años, 8-XII-1997, fl., G. Delucchi 1746 (LP), y General Alvarado: Miramar, zanja artificial alrededor del "Vivero Florentino Ameghino", 31-XII2005, fl., G. Charra 5 (LP).

\section{Discusión y Conclusiones}

Gladiolus dalenii fue introducida como ornamental en Misiones junto a otros gladiolos, en especial, los gladiolos de flores grandes. Estos últimos han sido, y son en la actualidad, ampliamente cultivados en zonas rurales, mayormente entre los descendientes de inmigrantes europeos. No ocurre lo mismo con $G$. dalenii, que no es llevada a ámbitos domésticos rurales por agricultores y chacareros locales, por temor a que se convierta en maleza, dado que se reconoce su potencial invasor. En las zonas periurbanas la situación es diferente porque los pobladores de escasos recursos la cultivan por su llamativa floración 
a partir de la extracción de bulbos de las plantas naturalizadas en bordes de caminos y rutas. Su presencia recurrente en estos "corredores" indica una posible expansión debida a la dispersión anemócora, facilitada por estas vías de acceso libres del obstáculo de la vegetación local. Esto se observa a menudo en Misiones para otras especies naturalizadas, cuyas diásporas son transportadas por las corrientes de aire. Por otro lado, las tareas de mantenimiento de rutas y caminos, como la limpieza de las cunetas (Eduardo A. Finkelstein, com. pers.), involucran movimientos de tierra que posibilitan la dispersión inadvertida de los cormos en los terrenos recién removidos.

El proceso de naturalización de especies que pueden convertirse en invasoras es entendido como un continuum dentro del cual una especie escapada de cultivo puede alcanzar la naturalización (como G. dalenii en la provincia de Misiones) o no (como los gladiolos híbridos de flores grandes encontrados en la provincia de Buenos Aires). Las especies naturalizadas establecen poblaciones que se autoperpetúan, se expanden e incorporan a la flora local sin intervención humana directa, al menos por diez años, sin comprometer la estabilidad de las comunidades nativas, que es el caso de las invasoras (Rejmánek, 2000; Richardson et al., 2000, 2010, 2011). En esta situación se halla en la actualidad $G$. dalenii en Misiones. Sus mecanismos de expansión en los sitios alterados donde se desarrollan sus poblaciones comprenden tanto la vía reproductiva (anemocoria), como la vegetativa (traslados de cormos en acarreos de tierra), mecanismo eficaz entre las herbáceas geófitas.

\section{Agradecimientos}

Los autores expresan su gratitud al personal de los herbarios CTES (Instituto de Botánica del Nordeste, IBONE-CONICET), y LP (Plantas Vasculares, Museo de La Plata), a Daniel H. Bazzano por el material fotográfico aportado, y a los revisores anónimos por sus oportunas sugerencias y comentarios.

\section{Bibliografía}

ANDERSON, E. W. \& R. PARK. 1989. Growing gladioli. Timber Press, Portland.

CANTOR, M. \& J. TOLETY. 2011. Gladiolus. En KOLE, C. (ed.), Wild crop relatives: genomic and breeding resources. Plantation and ornamental crops, pp. 133-159. Springer, Berlin. https://doi.org/10.1007/978-3-642-21201-7_8

DARLINGTON, C. D.\&A.P. WYLIE. 1955. Chromosome atlas of flowering plants. Allen \& Unwin, London.

DEHNEN-SCHMUTZ, K., J. TOUZA, C. PERRINGS \& M. WILLIAMSON. 2007. The horticultural trade and ornamental plant invasions in Britain. Conservation Biology 21: 224-231. https://doi.org/10.1111/j.1523-1739.2006.00538.x

DELUCCHI, G. \& J. A. HURRELL. 2009. Gladiolus. En HURRELL, J. A. (ed.), Flora Rioplatense 3 (4): 305-310. Editorial LOLA, Buenos Aires.

DIMITRI, M. J. 1987. Gladiolus. Enciclopedia Argentina de Agricultura y Jardinería 1 (1): 269271. Acme, Buenos Aires.

FACCIOLA, S. 1998. Cornucopia II. A source book of edible plants. Kampong, Vista.

FAWOLE, O. A., A. R. NDHLALA, S. O. AMOO, J. F. FINNIE \& J. VAN STADEN. 2009. Antiinflammatory and phytochemical properties of twelve medicinal plants used for treating gastrointestinal ailments in South Africa. Journal of Ethnopharmacology 123: 237-243.

https://doi.org/10.1016/j.jep.2009.03.012

FOTSING, D., G. T. NGOUPAYE, A. C. OUAFO, S. K. J. NJAPDOUNKE, Y. A. KENNETH \& E. N. BUM. 2017. Effects of Gladiolus dalenii on the stress-induced behavioral, neurochemical, and reproductive changes in rats. Frontiers in pharmacology 8: 685. https://doi.org/10.3389/fphar.2017.00685

FOXCROFT, L. C., D. M. RICHARDSON \& J. R. WILSON. 2008. Ornamental plants as invasive aliens: problems and solutions in Kruger National Park, South Africa. Environmental Management 41: 32-51.

https://doi.org/10.1007/s00267-007-9027-9

FRANCK, A. R., L. C. ANDERSON, J. R. BURKHALTER \& S. DICKMAN. 2016. Additions to the Flora of Florida, USA (2010-2015). Journal of the Botanical Research Institute of Texas 10: 175-190.

GBADAMOSI, I. T. 2012. Evaluation of antibacterial activity of six ethnobotanicals used in the treatment of infectious diseases in Nigeria. Botany Research International 5: 83-89.

GBADAMOSI, I. T. \& A. EGUNYOMI. 2014. Ethnobotanical survey of plants used for the treatment and management of sexually transmitted infections in Ibadan, Nigeria. Ethnobotany Research \& Applications 12: 659-669.

https://doi.org/10.17348/era.12.0.659-669 
GIORDANI, C., R. SANTIN \& M. B. CLEFF. 2015. Levantamento de extratos vegetais com ação anti-Candida no período de 2005-2013. Revista Brasileira de Plantas Medicinais 17: 175-185. https://doi.org/10.1590/1983-084X/12_072

GOLDBLATT, P. 1971. Cytological and morphological studies in the Southern African Iridaceae. Journal of South African Botany 37: 317-460.

GOLDBLATT, P. 1989. Systematics of Gladiolus L. (Iridaceae, Ixioideae) in Madagascar. Adansonia 3: 235-255.

GOLDBLATT, P. 1991. Iridaceae. En HUMBERT, H. (ed.), Flore de Madagascar et des Comores 45: 3-45. Muséum National d'Histoire Naturelle, Paris.

GOLDBLATT, P. 1993. Iridaceae. En POPE, G. V. (ed.), Flora Zambesiaca 12 (4): 1-106. Flora Zambesiaca Managing Committee, London.

GOLDBLATT, P. 1996a. Iridaceae. En POLHILL, R. M. (ed.), Flora of Tropical East Africa. A. A. Balkema, Rotterdam.

GOLDBLATT, P. 1996b. Gladiolus in Tropical Africa. Timber Press, Portland.

GOLDBLATT, P. 2002. Gladiolus. En FLORA OF NORTH AMERICA EDITORIAL COMMITTEE (ed.), Flora of North America North of Mexico 26: 407-409. Oxford University Press, New York.

GOLDBLATT, P. \& J. C. MANNING. 1998. Gladiolus in Southern Africa. Fernwood Press, Cape Town.

GOLDBLATT, P. \& J. C. MANNING. 2002. Evidence for moth and butterfly pollination in Gladiolus (Iridaceae, Crocoideae). Annals of the Missouri Botanical Garden 89: 110-124. https://doi.org/10.2307/3298660

GOLDBLATT, P. \& J. C. MANNING. 2006. Radiation of pollination systems in the Iridaceae of subSaharan Africa. Annals of Botany 97: 317-344. https://doi.org/10.1093/aob/mcj040

GOLDBLATT, P., M. TAKEI \& Z. A. RAZZAQ. 1993. Chromosome cytology in tropical African Gladiolus (Iridaceae). Annals of the Missouri Botanical Garden 80: 461-470. https://doi.org/10.2307/2399794

GOLDBLATT, P., J. C. MANNING \& P. RUDALL. 1998. Iridaceae. En KUBITZKI, K. (ed.), The families and genera of vascular plants 3: 295-333. Springer, Berlin. https://doi.org/10.1007/978-3662-03533-7 37

GOLDBLATT, P., A. RODRIGUEZ, M. P. POWELL, T. J. DAVIES, J. C. MANNING, M. VAN DER BANK \& V. SAVOLAINEN. 2008. Iridaceae 'Out of Australasia'? Phylogeny, Biogeography, and Divergence Time Based on Plastid DNA Sequences. Systematic Botany 33: 495-508. https://doi.org/10.1600/036364408785679806

GOVAERTS, R. \& C. BARKER. 2018. World Checklist of Iridaceae. Royal Botanic Gardens, Kew [online]. Disponible: http://wcsp.science.kew.org/ (Consulta: 3-X-2018).
GROVES, R., R. BODEN \& M. LONSDALE. 2005. Jumping the Garden Fence: Invasive Garden Plants in Australia. CSIRO-WWF-Australia, Sydney.

HAMILTON, A. P. 2005. Gladiolus. En TUTIN, T. G., V. H. HEYWOOD, N. A. BURGES, D. M. MOORE, D. H. VALENTINE, S. M. WALTERS \& D. A. WEBB (eds.), Flora Europaea 5: 101-102. $7^{\text {th }}$ printing. Cambridge University Press, Cambridge.

HEALY, A. J. \& E. EDGAR. 1980. Flora of New Zealand III. Government Printer, Wellington.

HOSKING, J. J., B. J. CONN, B. J. LEPSCHI \& C. H. BARKER. 2011. Plant species first recognised as naturalised or naturalising for New South Wales in 2004 and 2005. Cunninghamia 12: 85-114.

HURRELL, J. A. \& G. DELUCCHI. 2005. Iridaceae Ixioideae adventicias en la Argentina. Boletín de la Sociedad Argentina de Botánica 40: 289-296.

HURRELL, J. A. \& G. DELUCCHI. 2007 a. Amaryllidaceae adventicias en la Argentina. Boletín de la Sociedad Argentina de Botánica 42: 313-319.

HURRELL, J. A. \& G. DELUCCHI. $2007 \mathrm{~b}$. Agapanthaceae, Anthericaeae y Hyacinthaceae (Monocotyledoneae-Asparagales) adventicias en la Argentina. Revista del Museo Argentino de Ciencias Naturales (n. s.) 9: 103-107. https://doi.org/10.22179/REVMACN.9.301

HURRELL, J.A., D. H. BAZZANO \& G. DELUCCHI. 2005. Biota Rioplatense X. Monocotiledóneas herbáceas, nativas y exóticas. Editorial LOLA, Buenos Aires.

HURRELL, J. A., G. DELUCCHI \& F. BUET COSTANTINO. 2009. Nuevas citas de monocotiledóneas adventicias para la Argentina. Revista del Museo Argentino de Ciencias Naturales (n. s.) 11: 131-144. https://doi.org/10.22179/REVMACN.11.256

HURRELL, J. A., G. DELUCCHI \& J. A. TOLABA. 2010. Presencia de Lilium longiflorum (Liliaceae) adventicia en la Argentina. Boletín de la Sociedad Argentina de Botánica 45: 195-200.

HURRELL, J. A., G. DELUCCHI \& H. A. KELLER. 2019. Lilium regale (Liliaceae) naturalizada en la Argentina y nuevos registros para Brasil austral. Bonplandia 28: 71-75. https://doi.org/10.30972/bon.2813574

HYDE, M. A., B. T. WURSTEN, P. BALLINGS \& M. COATES PALGRAVE. 2019. Flora of Zimbabwe. genus page: gladiolus. Disponible: https://www.zimbabweflora.co.zw/speciesdata/ genus.php?genus_id=389 (Consulta: 12-II-2019).

KALAISELVI, S. 2002. Karyomorphological studies in varieties of Gladiolus primulinus and Tigridia pavoniana. Proceedings of the Indian Science Congress Association 87 (3, IV): 71-72.

KOWARIK, I. 2005. Urban ornamentals escaped from cultivation. En GRESSEL, J. (ed.), Crop ferality and volunteerism, pp. 97-121. CRC Press, Boca Raton. https://doi.org/10.1201/9781420037999.ch7 
LAWIN, I. F., F. O. LALÈYÈ, O. P. AGBANI \& A. E. ASSOGBADJO. 2015. Ethnobotanical assessment of the plant species used in the treatment of diabetes in the Sudano-Guinean zone of Benin. Journal of Animal and Plant Sciences 26: 4108-4123.

LIM, T. K. 2014. Edible Medicinal and Non Medicinal Plants. 8, Flowers. Springer, Dordrecht. https://doi.org/10.1007/978-94-017-8748-2

MUKHOPADHYAY, A. 1995. Gladiolus. Indian Council of Agricultural Research. New Delhi.

NGOUPAYE, G. T., E. N. BUM \& W. M. U. DANIELS. 2013a. Antidepressant-like effects of the aqueous macerate of the bulb of Gladiolus dalenii Van Geel (Iridaceae) in a rat model of epilepsy-associated depression. BMC Complementary and Alternative Medicine 13: 272.

https://doi.org/10.1186/1472-6882-13-272.

NGOUPAYE, G. T., E. N. BUM, E. NGAH, E. TALlA, F. C. U. MOTO, G. S. TAIWE, A. RAKOTONIRINA, S. V. RAKOTONIRINA. 2013b. The anticonvulsant and sedative effects of Gladiolus dalenii extracts in mice. Epilepsy \& Behavior 28: 450-456.

https://doi.org/10.1016/j.yebeh.2013.06.014

NGOUPAYE, G. T., E. N. BUM, G. S. TAIWE, F. C. O. MOTO \& E. TALLA. 2014. Antidepressant properties of aqueous macerate from Gladiolus dalenii corms. African Journal of Traditional, Complementary and Alternatives Medicines 11: 5361. https://doi.org/10.4314/ajtcam.v11i1.7

NGOUPAYE, G. T., D. B. PAHAYE, J. NGONDI, F. C. O. MOTO \& E. N. BUM. 2017. Gladiolus dalenii lyophilisate reverses scopolamine-induced amnesia and reduces oxidative stress in rat brain. Biomedicine and Pharmacotherapy 91: 350-357.

https://doi.org/10.1016/j.biopha.2017.04.061

ODHIAMBO, J. A., G. M. SIBOE, C. W. LUKHOBA \& S. F. DOSSAJI. 2010. Antifungal activity of crude extracts of Gladiolus dalenii Van Geel (Iridaceae). African Journal of Traditional, Complementary and Alternatives Medicines 7: 53-58.

https://doi.org/10.4314/ajtcam.v7i1.57254

ODHIAMBO, J. A., C. W. LUKHOBA \& S. F. DOSSAJI. 2011. Evaluation ofherbs as potential drugs/medicines. African Journal of Traditional, Complementary and Alternatives Medicines 8 (S): 144-151. https://doi.org/10.4314/ajtcam.v8i5S.20

OPIO, D. R., E. ANDAMA \& G. T. KUREH. 2017. Ethnobotanical survey of antimalarial plants in areas of: Abukamola, Angeta, Oculokori and Omarari, of Alebtong District in Northern Uganda. European journal of medicinal plants 21: 1-14. https://doi.org/10.9734/EJMP/2017/38043

PULLAIAH, T., K. V. KRISHNAMURTHY \& B. BAHADUR. 2016. Ethnobotany of India. 2. Western Ghats and West Coast of Peninsular India. CRC Press, Boca Raton.
PYŠEK, P. \& D. M. RICHARDSON. 2006. The biogeography of naturalization in alien plants. Journal of Biogeography 12: 2040-2050. https://doi.org/10.1111/j.1365-2699.2006.01578.x

PYŠEK, P., J. SADLO \& B. MANDÁK. 2002. Catalogue of alien plants of the Czech Republic. Preslia 74: 97-186.

PYŠEK, P., D. M. RICHARDSON, M. REJMÁNEK, G. L. WEBSTER, M. WILLIAMSON \& J. KIRSCHNER. 2004. Alien plants in checklists and floras: towards better communication between taxonomists and ecologists. Taxon 53: 131-143. https://doi.org/10.2307/4135498

QUATTROCCHI, U. 2016. CRC World Dictionary of Medicinal and Poisonous Plants. CRC Press, Boca Raton.

RANDALL, R. P. 2017. A Global Compendium of Weeds. 3rd ed. R. P. Randall, Perth.

REICHARD, S. H. \& P. WHITE. 2001. Horticulture as a pathway of invasive plant introductions in the United States. Bioscience 51: 103-113. https://doi. org/10.1641/0006-3568(2001)051[0103:HAAPOI]2. $0 . \mathrm{CO} ; 2$

REJMÁNEK, M. 2000. Invasive plants: approaches and predictions. Austral Ecology 25: 497-506. https://doi.org/10.1046/j.1442-9993.2000.01080.x

RICHARDSON, D. M. \& P. PYŠEK. 2012. Naturalization of introduced plants: ecological drivers of biogeographical patterns. New Phytologist 196: 383-396. https://doi. org/10.1111/j.1469-8137.2012.04292.x

RICHARDSON, D. M., P. PYŠEK, M. REJMÁNEK, M. G. BARBOUR, F. DANE PANETTA \& C. J. WEST. 2000. Naturalization and invasion of alien plants: concepts and definitions. Diversity and Distributions 6: 93-107. https://doi.org/10.1046/j.1472-4642.2000.00083.x

RICHARDSON, D. M., C. C. DAEHLER, M. R. LEISHMAN, A. PAUCHARD \& P. PYŠEK. 2010. Plant invasions: theoretical and practical challenges. Biological Invasions 12: 3907-3911. https://doi.org/10.1007/s10530-010-9845-1

RICHARDSON, D. M., P. PYŠEK \& J. T. CARLTON. 2011. A compendium of essential concepts and terminology in invasion ecology. En RICHARDSON, R. M. (ed.), Fifty Years of Invasion Ecology: The Legacy of Charles Elton, pp. 409-420. Blackwell, Chichester. https://doi.org/10.1002/9781444329988.ch30

THE PLANT LIST. 2013. The Plant List. Version 1.1. Royal Botanic Gardens, Kew, Missouri Botanical Garden [online]. Disponible: http://www.theplantlist.org/. (Consulta: 19-X-2018).

TROMBULAK, S. \& C. FRISSELL. 2000. Review of ecological effects of roads on terrestrial and aquatic communities. Conservation Biology 14: 18-30. https://doi.org/10.1046/j.1523-1739.2000.99084.x

TROPICOS. 2018. Tropicos.org, Missouri Botanical Garden. Disponible: http://www.tropicos.org (Consulta: 11-XI-2018).

SHARAIBI, O. J., D. A. ADEOGUN \& O. T. ABATI. 2017. Ethno-gynaeacological knowledge and 
preliminary phytochemical screenings of medicinal plants used in Lagos State, Nigeria. International Journal of Medicinal Plants and Natural Products 3: 6-18. https://doi.org/10.20431/2454-7999.0302002

SINGH, N., K. S. MAHAR, S. VERMA, B. MEENA, R. K. ROY, S. K. TEWARI, A. K. GOEL \& T. S. RANA. 2018. Molecular analysis of genetic variability and relationship among Gladiolus cultivars. Indian Journal of Biotechnology 17: 118-127.
VALENTE, L. M., J. C. MANNING, P. GOLDBLATT \& P. VARGAS. 2012. Did pollination shifts drive diversification in Southern African Gladiolus? Evaluating the model of pollinator-driven speciation. The American Naturalist 180: 83-98. https://doi.org/10.1086/666003

YEO, P. P. 2011. Gladiolus. En CULLEN, J., S. G. KNEES \& H. S. CUBEY (eds.). The European Garden Flora 1: 298-301. 2nd ed. Cambridge University Press, Cambridge. 